\title{
Study on Specific Combining Ability in Upland Cotton (G. hirsutum)
}

\author{
Dipali Ghive*, B.R. Patil, R.B. Ghorade and D.B. Dhumale \\ Dr PDKV Krishi Nagar Akola, India \\ *Corresponding author
}

\begin{abstract}
A B S T R A C T
Keywords

Cotton, Specific combining ability, 'A' and 'D' genome

Article Info

Accepted:

17 June 2018

Available Online:

10 July 2018

Ten genetically diverse parental lines were crossed in diallel fashion (excluding reciprocals). Ten parental lines, forty five hybrids and two checks were studied in kharif, 2009. Observations were recorded on fifteen characters viz., days to 50 per cent flowering, Days to maturity, Days to first boll bursting, plant height $(\mathrm{cm})$, number of monopodia per plant, number of sympodia per plant, number of bolls per plant, boll weight ( $\mathrm{g}$ ), seed cotton yield $(\mathrm{Kg} / \mathrm{ha})$, seed index $(\mathrm{g})$, lint yield $(\mathrm{kg} / \mathrm{ha})$, ginning out turn $(\%), 2.5$ per cent span length $(\mathrm{mm})$, micronaire value $(\mu \mathrm{g} / \mathrm{inch})$, fibre strength $(\mathrm{g} / \mathrm{tex})$, and uniformity ratio. Highest positively significant sca effect for seed cotton yield was recorded by the hybrid AKH 08-22 x BGP Sel SPS-18 followed by AKH 08-22 x IET SPS-2 and IET SPS-2 $x$ BBP Sel SPS-30.The hybrid AKH 08-22 x IET SPS-2 ranked first seed cotton yield and it exhibited significant sca effects in desirable direction for 2.5 per cent span length, micronaire value, uniformity ratio and other plant parameters like days to $50 \%$ flowering, days to first boll bursting, number of sympodia, number of bolls per plant, lint yield (kg/ha).The hybrid AKH 08-22 x BGP Sel SPS-18 recorded highest sca for yield and sca is desirable for micronaire value, fibre strength and uniformity ratio.
\end{abstract}

\section{Introduction}

Cotton belongs to the genus Gossypium which is one among eight genera under tribe gossypieae family malvaceae. This dicotyledonous genus comprises approximately 50 species of which 45 are diploid and five are allotetraploid. The lint bearing species of the genus Gossypium (the true cotton) are four out of which the diploid $(2 \mathrm{n}=26)$ species are $G$. arboreum $L$. and $G$. herbaceum $L$. which are indigenous to Asia and Africa and are popularly known as desi cotton in India. The new world cotton i.e. the tetraploid $(2 \mathrm{n}=52)$ species $G$. hirsutum $L$. and $G$. barbadense $L$. were initially introduced in India during the $17^{\text {th }}$ and $18^{\text {th }}$ century A.D. It has been shown that the new world cottons are natural amphidiploid containing the 'A' genome from Asiatic group (G. arboreum $L$. and G. herbaceum L.) and 'D' genome from a taxon of the American diploid group ( $G$. raimondii $L$.). The new world cottons are popularly known as American (G. hirsutum L.) and Egyptian (G.barbadense L.) cottons. Cotton is grown in about 111 countries of the world and Russia, USA, China, India, Brazil, Pakistan, Turkey and Egypt are the important cotton producing countries contributing 85 per 
cent of total world production. During the year 2009-2010 area under cotton in India was 101.12 lakh hectare with 285 lakh bales production and lint productivity of $525 \mathrm{~kg} / \mathrm{ha}$. In Maharashtra, area was 35.05 lakh hectare with 67 lakh bales production and productivity of lint was $325 \mathrm{~kg} / \mathrm{ha}$. In Vidarbha area was 13.15 lakh hectare with 24 lakh bales production and productivity of $311 \mathrm{~kg} / \mathrm{ha}$.

\section{Materials and Methods}

Ten genetically diverse parental lines were crossed in diallel fashion (excluding reciprocals). Ten parental lines, forty five hybrids and two checks were studied in kharif, 2009. Observations were recorded on fifteen characters viz; days to 50 per cent flowering, Days to maturity, Days to first boll bursting, plant height $(\mathrm{cm})$, number of monopodia per plant, number of sympodia per plant, number of bolls per plant, boll weight (g), seed cotton yield $(\mathrm{Kg} / \mathrm{ha})$, seed index (g), lint yield $(\mathrm{kg} / \mathrm{ha})$, ginning out turn (\%),2.5 per cent span length $(\mathrm{mm})$, micronaire value ( $\mu \mathrm{g} / \mathrm{inch})$, fibre strength $(\mathrm{g} / \mathrm{tex})$, and uniformity ratio.

The genetic analysis was carried out as per model I, method II of Griffing (1956). Heterosis was estimated over mid parent, better parent, standard hybrid PKV Hy-2 and PKV Hy-5. Results are briefly summarized below.

The most heterotic crosses over mid parent, better parent, standard hybrid for seed cotton yield per plant were AKH 08-22 x IET SPS-2, IET-2 x AKH-9913 and IET-2 x IET SPS-2 respectively. These hybrids also recorded highest seed cotton yield. The most heterotic crosses for important fibre properties were AKH08-22xAKH-9913 and AKH08-22 x AKH-9912 for 2.5 per cent span length, AKH 08-22 x IET-SPS-2 and AKH 08-22x BBP LS-43 for fibre strength

Results and Discussion

\section{Specific combining ability}

In crop improvement programme specific combining ability is important to pinpoint specific cross combination for commercial exploitation. Sca effects are indicative of heterosis. The top ten crosses exhibiting best per se performance for seed cotton yield are presented in Table 1 with their sca effects.

Highest positively significant sca effect for seed cotton yield per plant was observed in the cross AKH08-22 x BGP Sel SPS-18. Its rank was first in mean performance for seed cotton yield. This cross also exhibited significant positive sca effects for days to $50 \%$ flowering, days to first boll bursting, number of sympodia, number of bolls per plant, seed cotton yield $(\mathrm{kg} / \mathrm{ha})$, lint yield, micronaire value $(\mu \mathrm{g} /$ inch) fibre strength, uniformity ratio.

Another cross AKH 08-22 x IET SPS-2, recorded positively significant sca effects for seed cotton yield. This cross also recorded significant sca effects in desirable directions for days to 50 per cent flowering, days to first boll bursting, number of sympodia per plant, number of bolls per plant, boll weight, lint yield and $2.5 \%$ span length. Its sca effects for 2.5 per cent and micronaire value, fibre strength and uniformity were non significant but in desirable direction.

Nadarajan and Rangaswami (1990b) reported that the superiority of the hybrid appear to be not dependent on gca effects of the parents, sca effects of the hybrid and heterosis percentage of the hybrid. Only per se performance of the hybrid appears to be best criteria to fix an appropriate hybrid combination. Such findings were also reported by Tomar and Singh (1992) and Modi et al., (1999). However, Sambamurthy and Rangamadhacharyulu (1998), Ahuja and 
Tuteja (2000), Nageshwar Rao and Shiva Shantha Reddy (2001) observed close relationship between per se performance, combining ability effects and heterosis.

The other crosses which exhibited high desirable sca effects for component characters (Table 5.6 ) excluding yield were AKH08-22 $\mathrm{x}$ IET SPS-2 for days to 50 per cent flowering IET-2 x AKH-9913 for days to maturity, AKH 08-22 x BGP Sel SPS-18 for days to first boll bursting IET-2 x IET-SPS-2 for plant height, AKH 08-22 x IET-2 and IET SPS-2 x BBP Sel SPS-30 for number of monopodia per plant, AKH08-22 x BGP Sel SPS-18 and IET$2 \times$ AKH-9913 for number of sympodia per plant, AKH08-22 x BGP Sel SPS-18 and AKH08-22 x IET SPS-2 for number of bolls per plant, IET-2 x AKH-9913 and AKH 08-22 $\mathrm{x}$ AKH-9913 for boll weight. AKH 08-22 x IET-SPS-2 for seed cotton yield ( $\mathrm{kg} / \mathrm{ha})$, IET$2 \times$ AKH-9913 for seed index, AKH 08-22 x IET-2 and AKH08-22 x IET-2 for lint yield,
AKH 08-22 x IET-2 for ginning per cent

For fibre parameters, crosses which have exhibited high desirable sca effects (Table 5.6) were AKH 08-22 x AKH-9912 and IET-2 x IET SPS-2 for 2.5 per cent span length and IET-2 x IET-SPS-2 for micronaire value, AKH08-22 x BGP Sel SPS-18 forfibre strength and AKH-9913 x MCU-5VT for uniformity ratio exhibited desirable sca effects.

Crosses exhibiting high desirable sca effects for component characters excluding yield are practically of no use in general plant breeding programme, but can be used for the improvement of those characters insofar.

Abro (2009) reported crosses Sadori x CIM448 and Sadori x CRIS-134 exhibited highest sca effects for boll number/plant.

Table.1 Characteristics of parental lines

\begin{tabular}{|c|l|l|}
\hline Sr.No. & \multicolumn{1}{|c|}{ Parents } & \multicolumn{1}{|c|}{ Important features } \\
\hline $\mathbf{1}$ & AKH-08-22 & High yielding, long staple length, high ginning \\
\hline $\mathbf{2}$ & IET-2 & Medium tall, short sympodia, compact plant \\
\hline $\mathbf{3}$ & BGP Sel SPS-18 & Tall, big boll, medium duration \\
\hline $\mathbf{4}$ & IET-SPS-2 & Dense hairy leaves, bigboll, resistant to sucking \\
\hline $\mathbf{5}$ & AKH-9913 & High yielder, good bearing, medium duration \\
\hline $\mathbf{6}$ & MCU-5VT & Long staple \\
\hline $\mathbf{7}$ & AKH-9912 & Medium tall, long staple length \\
\hline $\mathbf{8}$ & BGP Sel SPS-4 & Tall, high yielder, long staple \\
\hline $\mathbf{9}$ & BBP Sel SPS-30 & High yielding, long staple length with good \\
\hline $\mathbf{1 0}$ & BBP LS-43 & Hairy, bigboll, short sympodia \\
\hline
\end{tabular}

Table. 2 Top ranking parents, best general combiners and $\mathrm{F}_{1 \mathrm{~s}}$ having high sca effects for different characters

\begin{tabular}{|c|c|c|c|c|c|}
\hline S.N. & Character & Best parent per se & $\begin{array}{l}\text { Best general } \\
\text { combiner in } \mathrm{F}_{1}\end{array}$ & Best $F_{1 s}$ per se & $\mathrm{F}_{1 \mathrm{~s}}$ showing high sca \\
\hline 1. & $\begin{array}{l}\text { Days to } \\
50 \% \\
\text { Flowering }\end{array}$ & $\begin{array}{l}\text { IET-2 (65.00) } \\
\text { BGP Sel SPS-4 } \\
(65.00)\end{array}$ & $\begin{array}{l}\text { BGP Sel SPS-18 } \\
(-2.022) \text { AKH } \\
08-22(-0.856)\end{array}$ & $\begin{array}{l}\text { AKH08-22 x IET } \\
\text { SPS -4(54.66) } \\
\text { BGP Sel SPS-18 x }\end{array}$ & $\begin{array}{l}\text { AKH08-22 x IET-2 } \\
(-7.75) \\
\text { IET SPS-2 x BBP }\end{array}$ \\
\hline
\end{tabular}




\begin{tabular}{|c|c|c|c|c|c|}
\hline & & $\begin{array}{l}\text { BGP Sel SPS-18 } \\
(65.67)\end{array}$ & $\begin{array}{l}\text { AKH-9913 (- } \\
0.383))\end{array}$ & $\begin{array}{l}\text { MCU - 5VT(57.33) } \\
\text { AKH08-22 x BGP } \\
\text { Sel SPS-4 (62.33) }\end{array}$ & $\begin{array}{l}\text { Sel SPS-30 (-3.45) } \\
\text { AKH08-22 x AKH- } \\
9912(-3.31)\end{array}$ \\
\hline 2. & $\begin{array}{l}\text { Days to } \\
\text { maturity }\end{array}$ & $\begin{array}{l}\text { BBP LS-43 } \\
(180.00) \\
\text { BGP Sel SPS-4 } \\
(180.00) \\
\text { AKH-9913 } \\
(180.33)\end{array}$ & $\begin{array}{l}\text { IET-SPS-2 } \\
(-1.494) \\
\text { AKH08-22 } \\
(-0.828) \\
\text { AKH } 9913 \\
(-0.494)\end{array}$ & $\begin{array}{l}\text { AKH-9913 x BGP } \\
\text { Sel SPS-4 (176.33) } \\
\text { IET-SPS-2 x BBP } \\
\text { Sel SPS -30 } \\
(176.35) \\
\text { AKH-9913 x BBP } \\
\text { Sel SPS -30 } \\
(175.67)\end{array}$ & $\begin{array}{l}\text { AKH08-22 x AKH } \\
9912(-3.94) \\
\text { IET-2 x AKH-9913 } \\
(-3.94) \\
\text { MCU-5VT x BBP } \\
\text { LS-43 (-3.75) }\end{array}$ \\
\hline 3. & $\begin{array}{l}\text { Days to } \\
\text { first boll } \\
\text { bursting }\end{array}$ & $\begin{array}{l}\text { AKH-9912 (120) } \\
\text { BBP Sel SPS-30 } \\
(119) \\
\text { BGP Sel SPS } \\
4(119)\end{array}$ & $\begin{array}{l}\text { BGP Sel SPS-18 } \\
(-2.044) \\
\text { IET-2 (-0.628) } \\
\text { MCU-5VT } \\
(-0.128)\end{array}$ & $\begin{array}{l}\text { BGP Sel SPS -18 x } \\
\text { MCU-5VT (107) } \\
\text { BGP Sel SPS- } 18 \text { x } \\
\text { BGP Sel SPS- } \\
4(112) \\
\text { AKH08-22 x IET- } \\
\text { SPS-2 (113) }\end{array}$ & $\begin{array}{l}\text { BGP Sel SPS-18 x } \\
\text { IET-SPS-2 }(5.33) \\
\text { AKH08-22 x AKH- } \\
9913(4.39) \\
\text { BGP Sel SPS-18 x } \\
\text { BGP Sel SPS-4(3.22) }\end{array}$ \\
\hline 4. & $\begin{array}{l}\text { Plant } \\
\text { height }(\mathrm{cm})\end{array}$ & $\begin{array}{l}\text { IET-2 (111.33) } \\
\text { AKH08-22 } \\
(109.33) \\
\text { BBP Sel SPS-30 } \\
(108.67)\end{array}$ & $\begin{array}{l}\text { AKH-9913 } \\
(1.628) \\
\text { MCU-5VT } \\
(0.711) \\
\text { IET SPS-2 } \\
(0.517)\end{array}$ & $\begin{array}{l}\text { AKH } 9913 \times \text { BBP } \\
\text { LS }-43 \text { (115) } \\
\text { IET }-2 \times \text { MCU-5VT } \\
(114.33) \\
\text { IET-2 x AKH-9913 } \\
(113.67)\end{array}$ & $\begin{array}{l}\text { IET-2 } \times \text { IET-SPS-2 } \\
(6.28) \\
\text { IET-2 } \times \text { BGP Sel } \\
\text { SPS-18 }(5.97) \\
\text { AKH08-22 x BGP } \\
\text { Sel SPS-4 (5.78) }\end{array}$ \\
\hline 5.(i) & $\begin{array}{l}\text { Number of } \\
\text { monopodia/ } \\
\text { plant }\end{array}$ & $\begin{array}{l}\text { BBP Sel SPS-30 } \\
(2.73) \\
\text { AKH-9913 (3.27) } \\
\text { IET-SPS-2 (3.37) }\end{array}$ & $\begin{array}{l}\text { IET-2 (-0.103) } \\
\text { AKH 9912 } \\
(-0.075) \\
\text { MCU-5VT } \\
(-0.061)\end{array}$ & $\begin{array}{l}\text { IET-2 x IET-SPS-2 } \\
(2.43) \\
\text { IET-SPS- } 2 \text { x BBP } \\
\text { LS-43 (2.57) } \\
\text { AKH-9912 x BGP } \\
\text { Sel SPS -4 (2.57) }\end{array}$ & $\begin{array}{l}\text { AKH-9913 x BBP } \\
\text { LS-43 (-0.89) } \\
\text { IET-SPS-2 x MCU- } \\
\text { 5VT (1-0.86) } \\
\text { BBP Sel SPS-30 x } \\
\text { BBP LS-43 (-0.77) }\end{array}$ \\
\hline 5.(ii) & $\begin{array}{l}\text { Number of } \\
\text { sympodia } \\
\text { per plant }\end{array}$ & $\begin{array}{l}\text { MCU-5VT (11.60) } \\
\text { IET-SPS-2 (13.93) } \\
\text { BGP Sel SPS-4 } \\
(14.00)\end{array}$ & $\begin{array}{l}\text { AKH08- } \\
22(3.666) \\
\text { IET-2 (0.769) }\end{array}$ & $\begin{array}{l}\text { AKH08-22 x IET-2 } \\
(25.33) \\
\text { AKH-08-22 x IET } \\
\text { SPS-2 (24.06) } \\
\text { AKH08-22 x BGP } \\
\text { SelSPS-30 (20-93) }\end{array}$ & $\begin{array}{l}\text { AKH08-22 x BGP } \\
\text { Sel SPS-18 (4.87) } \\
\text { IET-2 x AKH-9913 } \\
(2.31) \\
\text { AKH-08-22 x BGP } \\
\text { Sel SPS-4(2.27)) }\end{array}$ \\
\hline 6. & $\begin{array}{l}\text { Number of } \\
\text { bolls per } \\
\text { plant }\end{array}$ & $\begin{array}{l}\text { AKH } 9913(16.60) \\
\text { BGP SelSPS-4 } \\
(15.33) \\
\text { BBP LS-43 (11.93) }\end{array}$ & $\begin{array}{l}\text { AKH08-22 } \\
(3.274) \\
\text { IET-2 (2.227) } \\
\text { BGP Sel SPS-18 } \\
(0.718)\end{array}$ & $\begin{array}{l}\text { AKH08.22 x IET x } \\
\text { SPS-2 (27.40) } \\
\text { IET-2 x IET-SPS-2 } \\
(22.13) \\
\text { IET-2 x IET -SPS-2 } \\
(22.13)\end{array}$ & $\begin{array}{l}\text { AKH08-22 x BGP } \\
\text { Sel SPS-18 (8.71) } \\
\text { AKH08-22 } \times \text { IET- } \\
\text { SPS-2 }(6.29) \\
\text { IET-2 } \times \text { BGP Sel } \\
\text { SPS-18 (2.79) }\end{array}$ \\
\hline 7. & $\begin{array}{l}\text { Boll weight } \\
\text { (g) }\end{array}$ & $\begin{array}{l}\text { BBP Sel SPS-30 } \\
(3.77) \\
\text { BGP Sel SPS -18 }\end{array}$ & $\begin{array}{l}\text { MCU-5VT } \\
(0.223) \\
\text { IET-SPS-2 }\end{array}$ & $\begin{array}{l}\text { AKH-9913 x BBP } \\
\text { Sel SPS -30 (4.17) } \\
\text { IET-SPS-2 } \times \text { BBP }\end{array}$ & $\begin{array}{l}\text { MCU -5VT x BGP } \\
\text { Sel SPS-4 }(0.84) \\
\text { IET-SPS-2 } \times \text { BGP }\end{array}$ \\
\hline
\end{tabular}




\begin{tabular}{|c|c|c|c|c|c|}
\hline & & $\begin{array}{l}(3.17) \\
\text { BGP Sel SPS-4 } \\
(3.47)\end{array}$ & $\begin{array}{l}(0.154) \\
\text { AKH-9913 } \\
(0.095)\end{array}$ & $\begin{array}{l}\text { LS-43 (4.17) } \\
\text { IET-SPS-2 x AKH- } \\
9912(3.83)\end{array}$ & $\begin{array}{l}\text { Sel SPS-4 }(0.78) \\
\text { BGP Sel SPS-18 x } \\
\text { BGP Sel SPS-30 } \\
(0.75)\end{array}$ \\
\hline 8. & $\begin{array}{l}\text { Seed cotton } \\
\text { yield per } \\
\text { plant } \\
(\mathrm{kg} / \mathrm{ha})\end{array}$ & $\begin{array}{l}\text { AKH08-22 } \\
(1687.66) \\
\text { AKH-9913 } \\
(1562.33) \text { BGP Sel } \\
\text { SPS -4 (1465.33) }\end{array}$ & $\begin{array}{l}\text { AKH 08-22 } \\
(269.189) \\
\text { IET-2 (146.244) } \\
\text { BGP Sel SPS- } \\
18()\end{array}$ & $\begin{array}{l}\text { AKH X IET - SPS- } \\
(2555) \\
\text { IET-2 x AKH } 9913 \\
(2056) \\
\text { IET-2 x IET -SPS - } \\
2(2001)\end{array}$ & $\begin{array}{l}\text { AKH 08-22 x BGP } \\
\text { Sel SPS -18 (741-25) } \\
\text { IET -2 x BGP Sel } \\
\text { SPS-18 (365.17) } \\
\text { IET-SPS-2 x AKH- } \\
9913 \text { (33.109) }\end{array}$ \\
\hline 9. & ndex & $\begin{array}{l}\text { AKH -9912 (10.83) } \\
\text { AKH-9913 (10.50) } \\
\text { BBP LS-43 (10.17) }\end{array}$ & $\begin{array}{l}\text { AKH } 08-22 \\
(0.818) \\
\text { AKH- } 9913 \\
(0.271) \\
\text { BGP Sel SPS-4 } \\
(0.123)\end{array}$ & $\begin{array}{l}\text { AKH 08-22 x BBP } \\
\text { Sel SPS -30 (11.50) } \\
\text { IET-SPS-2 x BBP } \\
\text { Sel SPS-30 (11.33) } \\
\text { AKH08-22 x MCU- } \\
5 \mathrm{VT}(11.33)\end{array}$ & $\begin{array}{l}\text { IET-2 x BGP Sel } \\
\text { SPS-18 (1.22) } \\
\text { IET-SPS-2 x AKH- } \\
9913(1.15) \\
\text { AKH08-22 x IET- } \\
2(1)\end{array}$ \\
\hline 10. & $\begin{array}{l}\text { Lint yield } \\
(\mathrm{g})\end{array}$ & $\begin{array}{l}\text { AKH08-22 (617) } \\
\text { AKH-9913 } \\
\text { (563.66) } \\
\text { BBP LS-30 } \\
(525.00)\end{array}$ & $\begin{array}{l}\text { AKH08-22 } \\
(104.96) \\
\text { IET-2 (60.87) } \\
\text { BGP Sel SPS-18 } \\
(11.29)\end{array}$ & $\begin{array}{l}\text { AKH08-22 x IET - } \\
\text { SPS-(924.33) } \\
\text { IET-2 x AKH- } \\
\text { 9913(771.33) } \\
\text { IET-2 x IET-SPS-2 } \\
(750.00)\end{array}$ & $\begin{array}{l}\text { BGP Sel SPS-18 x } \\
\text { BGP Sel SPS-4 } \\
(98.67) \text { AKH08-22 x } \\
\text { BBP Sel SPS-30 } \\
(83.89) \text { BGP Sel } \\
\text { SPS-18 x BBP LS-43 } \\
(75.09)\end{array}$ \\
\hline 11. & $\begin{array}{l}\text { ing } \\
\text { urn }\end{array}$ & $\begin{array}{l}\text { AKH08-22 (36.53) } \\
\text { IET-2 (36.47) } \\
\text { BBP Sel SPS-30 } \\
(36.27)\end{array}$ & $\begin{array}{l}\text { IET - SPS-2 } \\
(0.558) \\
\text { AKH08-22 } \\
(0.492) \\
\text { IET-2 }(0.469)\end{array}$ & $\begin{array}{l}\text { IET-2 x BBP LS-43 } \\
(38.50) \\
\text { IET-SPS-2 x AKH- } \\
9913(38.80) \\
\text { AKH08-22 x BBP } \\
\text { LS-43 (38.40) }\end{array}$ & $\begin{array}{l}\text { BGP Sel SPS-18 x } \\
\text { BGP Sel SPS-4 } \\
(3.14) \\
\text { AKH08-22 x BBP } \\
\text { Sel SPS-30 }(2.24) \\
\text { IET-2 x BGP Sel } \\
\text { SPS-4 }(2.00)\end{array}$ \\
\hline 12. & $\begin{array}{l}\text { \% Span } \\
\text { gth }\end{array}$ & $\begin{array}{l}\text { BGP Sel SPS-4 } \\
(31.33) \\
\text { BBP Sel SPS-30 } \\
(31.06) \\
\text { IET-2(27.57) }\end{array}$ & $\begin{array}{l}\text { AKH-9913 } \\
(0.937) \\
\text { MCU. 5VT } \\
(0.651) \\
\text { BGP Sel SPS-18 } \\
(0.317)\end{array}$ & $\begin{array}{l}\text { MCU - 5 VT x } \\
\text { BBP LS-43 (32.23) } \\
\text { MCU-5VT X AKH } \\
-9912 \text { (31.9) } \\
\text { AKH08-22 X BGP } \\
\text { Sel SPS-4 (31.66) }\end{array}$ & $\begin{array}{l}\text { AKH08-22 x AKH - } \\
9912(2.39) \\
\text { IET-2 x IET-SPS-2 } \\
(2.26) \\
\text { AKH 08.22 x BGP } \\
\text { Sel SPS-4 (2.16) }\end{array}$ \\
\hline 13. & $\begin{array}{l}\text { Micronaire } \\
\text { value } \\
\text { (ug/inch) }\end{array}$ & $\begin{array}{l}\text { BGP Sel SPS-4 } \\
(3.60) \\
\text { AKH-9912 (3.60) } \\
\text { BBP LS-43 (4.13) }\end{array}$ & $\begin{array}{l}\text { AKH-9913 (- } \\
0.170) \\
\text { BGP Sel SPS-18 } \\
(-0.089) \\
\text { BBP Sel SPS-30 } \\
(-0.073)\end{array}$ & $\begin{array}{l}\text { BGP Sel SPS-18 x } \\
\text { MCU-5VT (3.46) } \\
\text { AKH08-22 x IET- } \\
\text { SPS-2 (3.73) } \\
\text { MCU-5VT x BBP } \\
\text { Sel SPS-30 (3.80) }\end{array}$ & $\begin{array}{l}\text { BGP Sel SPS-4 x } \\
\text { BBP LS-43 }(-0.71) \\
\text { IET-2 x IET - SPS-2 } \\
(-0.62) \\
\text { IET -2 X AKH-9912 } \\
(-0.51)\end{array}$ \\
\hline 14. & $\begin{array}{l}\text { Fibre } \\
\text { strength } \\
\text { (g/tex) }\end{array}$ & $\begin{array}{l}\text { BBP Sel SPS-30 } \\
(20.36) \\
\text { BGP Sel SPS-18 } \\
(20.10)\end{array}$ & $\begin{array}{l}\text { BGP Sel SPS- } 18 \\
(0.623) \\
\text { AKH08-22 } \\
(0.454)\end{array}$ & $\begin{array}{l}\text { BGP Sel SPS-18 x } \\
\text { MCU-5VT (23.90) } \\
\text { AKH08-22 x IET- } \\
\text { SPS-2 (22.40) }\end{array}$ & $\begin{array}{l}\text { AKH08-22 x BGP } \\
\text { Sel SPS-18 (1.37) } \\
\text { MCU-5VT x BBP } \\
\text { LS-43 (1.26) }\end{array}$ \\
\hline
\end{tabular}




\begin{tabular}{|c|c|c|c|c|c|}
\hline & & AKH9913 (20.00) & $\begin{array}{l}\text { AKH-9913 } \\
(0.093)\end{array}$ & $\begin{array}{l}\text { MCU-5VT x BBP } \\
\text { LS-43 (21.10) }\end{array}$ & $\begin{array}{l}\text { AKH-9913 x BBP } \\
\text { Sel SPS-30 (1.05) }\end{array}$ \\
\hline 15. & $\begin{array}{l}\text { Uniformity } \\
\text { ratio }\end{array}$ & $\begin{array}{l}\text { IET-SPS-2 (53.20) } \\
\text { BBP LS-43 (52.27) } \\
\text { AKH-9913 (52.10) }\end{array}$ & $\begin{array}{l}\text { IET-2 }(1.055) \\
\text { BGP Sel SPS - } \\
18(0.79) \\
\text { IET-SPS-2 } \\
(0.585)\end{array}$ & $\begin{array}{l}\text { IET-2 x BBP -LS- } \\
43(52.33) \\
\text { IET-2 } \times \text { BGP Sel } \\
\text { SPS-18 }(52.23) \\
\text { AKH08-22 } \times \text { BGP } \\
\text { Sel SPS-18 }(52.23)\end{array}$ & $\begin{array}{l}\text { BGP Sel SPS -18 x } \\
\text { AKH-9913 (2.38) } \\
\text { AKH-9912 x BBP } \\
\text { LS-43 (3.12) } \\
\text { IET- 2x MCU-SVT } \\
(3.8)\end{array}$ \\
\hline
\end{tabular}

It is concluded that the crosses AKH 08-22 x IET SPS-2 and IET-2 $x$ IET SPS-2 were best considering mean values, heterosis and combining ability effects of seed cotton yield and important fibre properties. The cross AKH 08-22 x IET SPS-2 and IET-2 x IET SPS-2 ranked first and third for seed cotton yield per plant. These two hybrids exhibited high heterosis over mid parent, better parent and checks for seed cotton yield per plant and important fibre properties. These crosses also possessed significant sca effects in desirable direction for seed cotton yield per plant and important fibre properties.

Remaining eight crosses were promising for seed cotton yield per plant as they recorded high means, high heterotic value and significant sca effects for seed cotton yield per plant but their performance for fibre properties was poor except AKH 08-22 x BGP Sel SPS-18 which ranked seventh for mean performance of seed cotton yield (kg/ha) and had shown highest sca effects among ten parents it had shown significant sca performance for micronaire value ( $\mu \mathrm{g} /$ inch) and fibre strength $(\mathrm{g} /$ tex $)$

The parental combination in above crosses was either high $\mathrm{x}$ high gca, low x high gca or high $x$ low gca. Therefore, it appeared that for getting good cross combinations at least one of the parent should have good gca.

Pavasia et al., (1990), Sambamurthy and Ranganadhcharyulu (1998) and Ahuja and Tuteja (1999) also reported similar observations.
Jagtap and Kolhe (1987) reported partial dominance for seed cotton yield, ginning out turn and halo length. Singh Sanyansi (1991) reported over dominance for seed cotton yield and partial dominance for boll number. Sayal and Sulemani (1996b) reported over dominance for lint percentage seed index and staple length; Khan et al., (1997) reported partial dominance for staple length. Partial dominance for fibre length has been reported by Nistor and Nistor (1999)

The value of ratio of proportion of dominant and recessive genes in the parents was greater than unity for the characters viz., plant height, number of monopodia per plant, boll weight, ginning percentage, 2.5 per cent span length, micronaire value, fibre strength and fibre elongation showing asymmetrical distribution of genes with minority of recessive alleles and excess of dominant alleles for these traits. However ratio was nearly equal to unity for the characters days to 50 per cent flowering, number of sympodia per plant, number of bolls per plant, seed index, uniformity ratio and seed cotton yield per plant indicating symmetrical distribution of dominant and recessive alleles in the parents for these characters.

\section{References}

Griffing, B. $1956^{\mathrm{a}}$. Concept of general and specific combining ability in relation to diallel crossing system. Aust. J. Biol. Sci. 2:463-493. 
Griffing, B. $1956^{\mathrm{b}}$. A generalised treatment of the use of diallel crosses in quantittive inheritance. Heredity. 10:31-50.

Nadarajan, N. and S. Rangasamy. $1990^{\mathrm{b}}$. Study of heterosis and combining ability in (Gossypium hirsutumL.). J. Indian Soc. Cotton Improv. 15(2): 8894.

Tomar, S.K. and S.P. Singh. 1992. Combining ability analysis over environments in asiatic cotton (Gossypium arboreumL.). Indian J. Genet. 52(3):264-269.

Modi, N.D.; U.G. Patel; J.C. Patel and A.I. Maisuria. 1999. General and specific combining ability for major yield components in diploid cotton. J. Indian Soc. Cotton Improv. 24(2):129-131.

Sambamurthy, J.S.V. and N. Ranganadhacharyulu. 1998. Combining ability studies in diallel crosses of cotton. Ann. agric. Res. 19(4):441-444.

Sambamurthy, J.S.V. and N. Ranganadhacharyulu. 1999. Diallel cross analysis for combining ability in upland cotton (Gossypium hirsutumL.), Madras Agric. J. 86(7-9): 409-414.

Ahuja, S.L. and O.P. Tuteja. 1999. Genetic analysis of some quantitative characters in cotton (Gossypium hirsutumL.). J. Indian Soc. Cotton Improv. 24 (3): 191193.

Ahuja, S.L. and O.P. Tuteja. 2000. Heterosis and combining ability for yield and its components traits in upland cotton. J. Cotton Res. Dev., 14 (2):138-142.

Nageshwar Rao, G. and Shiva Shantha Reddy. 2001. Heterosis and combining ability for yield and its components in upland cotton. J. Indian Soc. Cotton Improv. 26(2):67-73.

Abro, S., M.M. Kandhro, S. Loghari, M.A. Arain and Z.A. Deho (2009):Combining ability and heterosis for yield contributing traits in upland cotton $(G$. hirsutum L..) Pak. J. Bot., 41(4):17691774

Pavasia, M.J. and P.T. Shukla. 1997. Combining ability analysis for fibre characters over environments in upland cotton. J. Cotton Res. Dev. 11(2):148155.

Jagtap, D.R. and A.K. Kolhe. 1987. Graphical analysis of yield and its components in upland cotton (Gossypium hirsutumL.). J. Indian Soc. Cotton Improv. 12(2):7783.

\section{How to cite this article:}

Dipali Ghive, B.R. Patil, R.B. Ghorade and Dhumale, D.B. 2018. Study on Specific Combining Ability in Upland Cotton (G.hirsutum). Int.J.Curr.Microbiol.App.Sci. 7(07): 2478-2484. doi: https://doi.org/10.20546/ijcmas.2018.707.290 